\title{
RNAi-mediated knock-down of Dab and Numb attenuate $A \beta$ levels via $\gamma$-secretase mediated APP processing
}

\author{
Zhongcong Xie ${ }^{1,2^{*}}$, Yuanlin Dong ${ }^{1,2}$, Uta Maeda ${ }^{1,2,4}$, Weiming Xia ${ }^{3}$ and Rudolph E Tanzi ${ }^{i^{*}}$
}

\begin{abstract}
Amyloid- $\beta$-protein (AB), the key component of senile plaques in Alzheimer's disease (AD) brain, is produced from amyloid precursor protein (APP) by cleavage of $\beta$-secretase and then $\gamma$-secretase. APP adaptor proteins with phosphotyrosine-binding (PTB) domains, including Dab (gene: DAB) and Numb (gene: NUMB), can bind to and interact with the conserved YENPTY-motif in the APP C-terminus. Here we describe, for the first time, the effects of RNAi knock-down of Dab and Numb expression on APP processing and A $\beta$ production. RNAi knock-down of Dab and Numb in $\mathrm{H} 4$ human neuroglioma cells stably transfected to express either FL-APP (H4-FL-APP cells) or APP-C99 (H4-APP-C99 cells) increased levels of APP-C-terminal fragments (APP-CTFs) and lowered A $\beta$ levels in both cell lines by inhibiting $\gamma$-secretase cleavage of APP. Finally, RNAi knock-down of APP also reduced levels of Numb in H4-APP cells. These findings suggest that pharmacologically blocking interaction of APP with Dab and Numb may provide novel therapeutic strategies of AD. The notion of attenuating $\gamma$-secretase cleavage of APP via the APP adaptor proteins, Dab and Numb, is particularly attractive with regard to therapeutic potential, given that side effects of $\gamma$ secretase inhibition owing to impaired proteolysis of other $\gamma$-secretase substrates, e.g. Notch, might be avoided.
\end{abstract}

\section{Introduction}

Amyloid- $\beta$-protein $(A \beta)$, the key component of senile plaques in Alzheimer's disease (AD) neuropathology, was first isolated from meningovascular amyloid deposits in $\mathrm{AD}$ and Down's syndrome [1,2], and has also been reported to be the subunit of the plaque amyloid [2-4]. The current amyloid hypothesis of AD states that the imbalance between $A \beta$ generation and $A \beta$ clearance is the basis of $A D$ neuropathogenesis. $A \beta$ is generated from amyloid precursor protein (APP). Specifically, APP is first hydrolyzed by $\beta$-secretase to generate a 99 -residue membrane-associated C-terminus fragment (APPC99) [5-8]. APP-C99 is further cleaved to release a $\sim 4-$ $\mathrm{kDa}$ peptide, $\mathrm{A} \beta$, and the amyloid precursor protein intracellular domain (AICD). This cleavage is achieved by an unusual form of proteolysis in which the protein is cleaved within the transmembrane domain (at residue +40 or +42$)$ by $\gamma$-secretase [9-11]. $\alpha$-secretase cleaves

\footnotetext{
* Correspondence: zxie@partners.org; tanzi@helix.mgh.harvard.edu 'Department of Neurology, Genetics and Aging Research Unit, MassGeneral Institute for Neurodegenerative Disease, Massachusetts General Hospital and Harvard Medical School, Charlestown, MA 02129-2060, USA Full list of author information is available at the end of the article
}

the majority of APP in the middle of the A $\beta$ region of APP. This cleavage will preclude $A \beta$ generation, lead to the release of a large ectodomain ( $\alpha$-APPs), and leave behind a carboxy-terminus fragment of 83 amino acids (APP-C83) in the membrane. $\gamma$-Secretase cleaves APPC83 to produce $\mathrm{p} 3$, an amino-terminally truncated form of $A \beta[12,13]$, [see review in [14]].

The cleavage of the APP cytoplasmic tail by $\gamma$-secretase generates AICD, which contains the strongly conserved YENPTY-motif. The YENPTY-sequence is a consensus motif for the binding of adaptor proteins that possess a phosphotyrosine-binding domain (PTB) present in several APP adaptor proteins, such as X11, Fe65, ShcC, Numb, Dab and JIP families [see review in [15]]. We have previously reported that RNAi knock-down of $\mathrm{X} 11 \alpha$, ShcC and Fe65 in H4 human neuroglioma cells lower $A \beta$ levels $[16,17]$.

Dab (encoded by gene $D A B$ ), the PTB-containing APP adaptor protein, can bind to and interact with the YENPTY-motif of APP $[18,19]$. Dab has been reported to function as an adaptor molecule in signal transduction process $[20,21]$. Numb (encoded by gene $N U M B$ ) is known to interact via its PTB domain with APP [22,23].

\section{()


A recent study [24] also suggest that high levels of Notch, another substrate of $\gamma$-secretase, can reduce levels of Numb and Numblike.

To date, the effects of reduced expression of Dab and Numb on APP processing and A $\beta$ production, the key components of $\mathrm{AD}$ neuropathogenesis, have not been assessed. For this purpose, we established RNAi knockdown of Dab and Numb in H4 human neuroglioma cells overexpressing full-length (FL)-APP (H4-FL-APP cells) and C-99 (H4-APP-C99 cells), and evaluated the effects of RNAi-mediated knock-down of Dab and Numb on APP processing and $\mathrm{A} \beta$ levels.

\section{Experimental procedures Cell lines}

We employed naïve $\mathrm{H} 4$ human neuroglioma $(\mathrm{H} 4)$ cells and $\mathrm{H} 4$ cells stably transfected to express either FL-APP (H4-FL-APP cells) or APP-C99 (H4-APP-C99 cells). Peptide APP-C99 is the product of $\beta$-secretase, which therefore contains $\alpha$ - and $\gamma$-, but not $\beta$-cleavage sites. The H4-APP-C99 cells provide a valid system to assess whether any effects on APP processing are dependent on $\gamma$-secretase-mediated APP processing and independent of $\beta$-secretase-mediated APP processing. All cell lines were cultured in DMEM (high glucose) containing 9\% heat-inactivated fetal calf serum, 100 units $/ \mathrm{ml}$ penicillin, $100 \mu \mathrm{g} / \mathrm{ml}$ streptomycin, and $2 \mathrm{mM} \mathrm{L}$-glutamine. Stably transfected $\mathrm{H} 4$ cells were additionally supplemented with $200 \mu \mathrm{g} / \mathrm{ml} \mathrm{G} 418$.

\section{RNAi treatment}

Small interfering RNA (siRNA) duplex was designed and obtained from Qiagen against human $N U M B$, the gene encoding Numb (5'- CAGCCTCTTGACCTCGGATAA3'). Dab siRNA duplex for $D A B$, the gene encoding Dab, was designed and obtained from Dharmacon research, Inc. (Lafayette, CO 80026) (5'-NNAGGUCAGGAUCG CAGUGAA-3'). Scrambled siRNA (AATTCTCC GAACGTGTCACGT) was obtained from Qiagen and was used as the control siRNA. siRNAs were transfected into cells by using electroporation (AMAXA, Gaithersburg, MD). We mixed 1 million cells, 100 ul AMAXA electroporation transfection solution and $10 \mathrm{ul} 20 \mathrm{uM}$ siRNA together, then we employed C-9 program in the AMAXA electroporation device for the cell transfection. We chose 20 uM siRNA of $N U M B$ and $D A B$ in current studies because our previous studies have shown that 20 uM siRNA of other APP adaptor protein genes, including $A P B A 1$ [17], SHC3 [16] and APBB1 [16], can affect APP processing and reduce $A \beta$ levels. The transfected cells then were placed in one well of a six-well plate containing $1.5 \mathrm{ml}$ cell culture media. The cells were harvested 48 hours after siRNA treatments.

\section{Cell lysis and protein amount quantification}

Cell pellets were detergent-extracted on ice using immunoprecipitation buffer $(10 \mathrm{mM}$ Tris- $\mathrm{HCl}, \mathrm{pH}$ 7.4, 150 $\mathrm{mM} \mathrm{NaCl}, 2 \mathrm{mM}$ EDTA, 0.5\% Nonidet P-40) plus protease inhibitors $(1 \mu \mathrm{g} / \mathrm{ml}$ aprotinin, $1 \mu \mathrm{g} / \mathrm{ml}$ leupeptin, 1 $\mu \mathrm{g} / \mathrm{ml}$ pepstatin A). The lysates were collected, centrifuged at 12,000 rpm for $10 \mathrm{~min}$, and quantified for total proteins by the BCA protein assay kit (Pierce, Iselin, NJ).

\section{Western blot analysis of APP processing}

Western blot analysis was performed as described by Xie et al. [25]. Briefly, $40 \mu \mathrm{g}$ of total protein of each sample was subjected to SDS-polyacrylamide gel electrophoresis using 4-20\% gradient Tris/glycine gels under reducing conditions (Invitrogen, Carlsbad, CA). Next, proteins were transferred to a polyvinylidene difluoride membrane (Bio-Rad, Hercules, CA) using a semi-dry electrotransfer system (Amersham Biosciences, San Francisco, CA). Nonspecific proteins were blocked using $5 \%$ non-fat dry milk in TBST for $1.5 \mathrm{~h}$. Blots were then incubated with a primary antibody, followed by a secondary antibody (horseradish peroxidase-conjugated anti-rabbit antibody 1:10,000; Pierce, New York, NY). Blots were washed with TBST for 30 min between steps. Antibody Dab (1:1,000, Novus Biologicals, Littleton, CO) was used to recognize Dab (35 kDa), antibody Numb (1:1,000, Abcam, Cambridge, MA) was used to detect Numb (75 kDa). Antibody A8717 (1:1,000, Sigma, St. Louis, MO) was used to visualize FL-APP (110 kDa), APP-C83 (12 kDa) and APP-C99 (10 kDa) in the Western blot analysis. The intensity of signals was analyzed using an image program (NIH Image 1.62). We first used the levels of $\beta$-actin to normalize the levels of Numb, Dab, FL-APP and APP-CTFs (e.g., determining the ratio of Numb amount to $\beta$-actin amount) to control for loading differences in total protein amounts. We then presented the changes in the protein levels of Numb, Dab, FL-APP, APP-C99 and APP-C83 in the cells treated with Numb or Dab siRNA as the percentage of those in the cells treated with control siRNA.

\section{Quantitation of A $\beta$ using Sandwich ELISA assay}

Following the treatment with control siRNA, Numb siRNA or Dab siRNA, conditioned media was collected, and secreted $A \beta$ was measured with a Sandwich ELISA assay by the A $\beta$ ELISA Core Facility at the Center for Neurological Diseases, Harvard Institute of Medicine, Harvard Medical School, Boston, Massachusetts, as described by Xie et al. [16]. Specifically, 96-well plates were coated with mouse monoclonal antibodies $(\mathrm{mAb})$ specific to $A \beta 40$ (2 G3). Following blocking with Block Ace, wells were incubated overnight at $4^{\circ} \mathrm{C}$ with test samples of conditioned cell culture media, and then an 
anti-A $\beta$ ( $\alpha$-A $\beta$-HR1) conjugated to horseradish peroxidase was added. Plates were then developed with TMB reagent and well absorbance was measured at $450 \mathrm{~nm}$. $\mathrm{A} \beta$ levels in test samples were determined by comparison with the signal from unconditioned media spiked with known quantities of $\mathrm{A} \beta 40$.

\section{Statistics}

ANOVA with repeated measurements was employed to compare the difference from the control group. P-values less than 0.05 were considered statistically significant.

\section{Results}

RNAi knock-down of Dab increased APP-CTFs levels and decreased $A \beta$ levels in H4-FL-APP cells

We first established conditions under which RNAi knock-down of Dab significantly reduced protein levels of Dab in H4-FL-APP cells. The cells were harvested 48 hours after being transfected with either control siRNA or Dab siRNA, and were subjected to Western blot analyses in which antibody Dab was used to visualize Dab levels. Immunoblotting for Dab revealed a visible reduction in Dab levels following Dab siRNA treatment as compared to control siRNA treatment (Figure 1A). Dab siRNA treatment significantly reduced Dab levels by $44 \%$ (normalized to $\beta$-actin) as compared to control siRNA treatment (Figure $1 \mathrm{~B}, * \mathrm{p}<0.05$ ).

Next, we assessed effects of RNAi-mediated knockdown of Dab on APP processing in H4-FL-APP cells by measuring protein levels of FL-APP, APP-C99 and APPC83 following Dab or control siRNA treatments. 48 hours after transfection of Dab siRNA or control siRNA, the cells were harvested and subjected to Western blot analyses in which antibody A8717 was used to detect FL-APP, APP-C99 and APP-C83. Immunoblot analysis of APP-CTFs revealed increases in levels of APP-C99 and APP-C83 in the cells treated with Dab siRNA as compared to the cells treated with control siRNA (Figure 1A). Meanwhile, no significant differences in FLAPP levels were observed between Dab siRNA - and control siRNA-treated cells. Quantification of FL-APP, APP-C99 and APP-C83 (normalized to $\beta$-actin) revealed that Dab siRNA treatment led to a $207 \%$ increase in ratio of APP-C99 to FL-APP (Figure 1C, *p < 0.05), and a similar (176\%) increase in ratio of APP-C83 to FLAPP (Figure 1D, *p $<0.05$ ), as compared to control

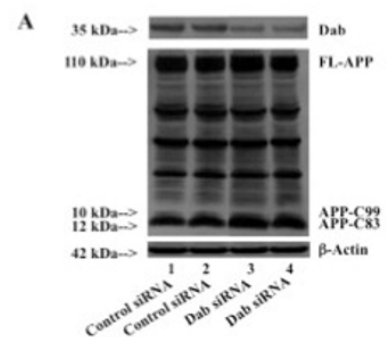

B

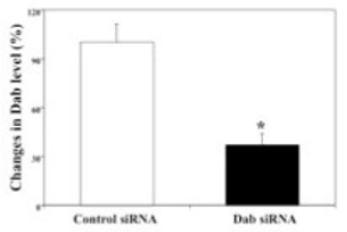

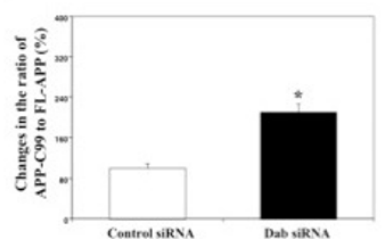

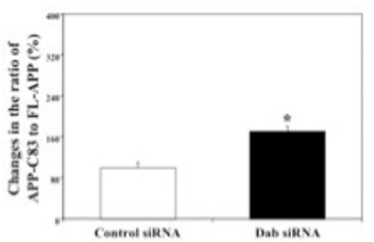

E

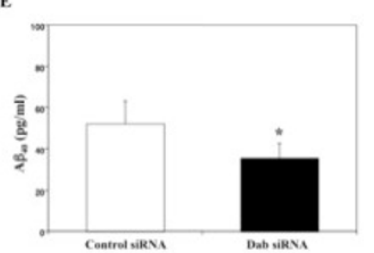

Figure 1 Effects of RNAi knock-down of Dab on APP processing and A $\boldsymbol{\beta}$ levels in H4-FL-APP cells. In H4-FL-APP cells, Dab siRNA treatment decreases protein levels of Dab, increases protein levels of APP-C83 and APP-C99, and decreases A $\beta$ levels. A. APP processing in Western blot analyses. Dab immunoblotting shows reductions in protein levels of Dab in the cells treated with Dab siRNA (columns 3 and 4) as compared to control siRNA (columns 1 and 2). FL-APP immunoblotting reveals that there is no significant difference in the protein levels of FLAPP in the cells treated with control siRNA or Dab siRNA. APP-CTFs immunoblotting shows increases in protein levels of APP-C99 and APP-C83 in the cells treated with Dab siRNA (columns 3 and 4) as compared to control siRNA (columns 1 and 2). B. Protein levels of Dab assessed by quantifying Dab in the Western blot. Dab siRNA treatment (black bar) significantly decreases protein levels of Dab as compared to control siRNA treatment (white bar) $\left({ }^{*} p<0.05\right)$, normalized to $\beta$-actin. C. APP processing assessed by quantifying ratio of APP-C99 to FL-APP in the Western blot. Dab siRNA treatment (black bar) significantly increases ratio of APP-C99 to FL-APP as compared to control siRNA treatment (white bar) ${ }^{*} \mathrm{P}$ $<0.05$ ), normalized to $\beta$-actin. D. APP processing assessed by quantifying ratio of APP-C83 to FL-APP in the Western blot. Dab siRNA treatment (black bar) significantly increases ratio of APP-C83 to FL-APP as compared to control siRNA treatment (white bar) $\left({ }^{*} p<0.05\right)$, normalized to $\beta$ actin. E. Effects of RNAi knock-down of Dab on A $\beta$ levels in H4-FL-APP cells. Dab siRNA treatment (black bar) decreases A 40 levels as compared to control siRNA treatment (white bar) $\left({ }^{*} p<0.05\right.$ ). 
siRNA treatment. Note the different parts of Western blot representing Dab were obtained from same samples of the experiments.

Next, we assessed effects of Dab siRNA on A $\beta$ levels in conditioned media. 48 hours after treatment with control siRNA or Dab siRNA, we measured secreted A $\beta 40$ levels in conditioned cell culture media. Dab siRNA treatment significantly decreased $A \beta 40$ levels as compared to control siRNA treatment (Figure 1E, " $\mathrm{p}<0.05$ ): $34 \mathrm{pg} / \mathrm{ml}$ (Dab siRNA) versus $57 \mathrm{pg} / \mathrm{ml}$ (control siRNA). Collectively, these data indicate that RNAi knock-down of Dab increases levels of APP-C99, APP-C83, and decreases secreted $\mathrm{A} \beta$ in H4-FL-APP cells, in a manner similar to that of $\gamma$-secretase inhibitor treatment.

RNAi knock-down of Numb increased APP-CTFs levels and decreased $A \beta$ levels in H4-FL-APP cells

We next assessed effects of RNAi knock-down of Numb, another APP adapter molecule, on APP processing and A $\beta$ levels in H4-FL-APP cells. We first established conditions under which Numb siRNA treatment reduced protein levels of Numb in H4-FL-APP cells. The cells were harvested 48 hours after being transfected with either control siRNA or Numb siRNA, and were subjected to Western blot analyses with Numb antibody to measure protein levels of Numb. Numb immunoblotting revealed a $54 \%$ reduction in protein levels of Numb following Numb siRNA treatment as compared to control siRNA treatment (Figure 2A, B, ${ }^{*} \mathrm{p}<0.05$ ).

We then assessed effects of RNAi-mediated knockdown of Numb on APP processing in H4-FL-APP cells by measuring levels of FL-APP, APP-C99 and APP-C83 following Numb siRNA or control siRNA treatments. 48 hours after transfection of Numb siRNA or control siRNA, the cells were harvested and subjected to Western blot analyses with antibody A8717 to detect FLAPP, APP-C99, and APP-C83. Levels of APP-C99 and APP-C83 were increased in the cells treated with Numb siRNA versus control siRNA (Figure 2A). Meanwhile, no significant differences in FL-APP levels were observed for Numb siRNA versus control siRNA-treated cells (Figure 2A). Numb siRNA treatment led to a $202 \%$ increase in ratio of APP-C99 to FL-APP (Figure 2C, * $\mathrm{p}<0.05)$, but only a mild $(122 \%)$ increase in ratio of APP-C83 to FL-APP (Figure 2D, N.S.), normalized to $\beta$-actin, as compared to control siRNA treatment.
A

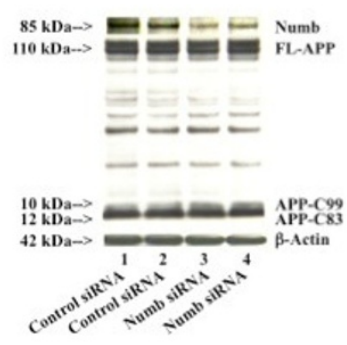

C

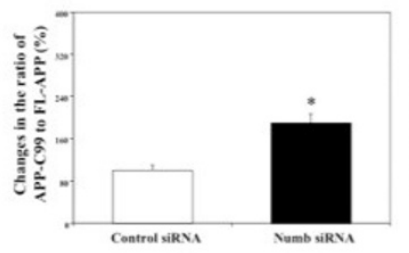

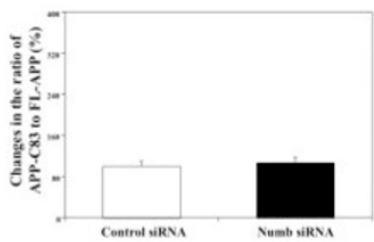

B

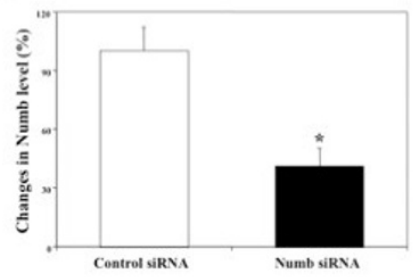

E

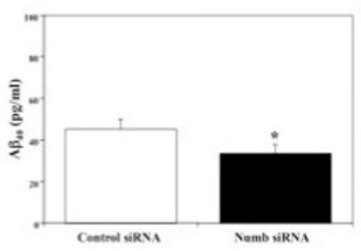

Figure 2 Effects of RNAi knock-down of Numb on APP processing and A $\boldsymbol{\beta}$ levels in H4-FL-APP cells. In H4-FL-APP cells, Numb siRNA treatment decreases protein levels of Numb, increases protein levels of APP-C99, and decreases A $\beta$ levels. A. APP processing in Western blot analyses. Numb immunoblotting shows reductions in protein levels of Numb in the cells treated with Numb siRNA (columns 3 and 4 ) as compared to control siRNA (columns 1 and 2). FL-APP immunoblotting reveals that there is no significant difference in protein levels of FL-APP in the cells treated with control siRNA or Numb siRNA. APP-CTFs immunoblotting shows increases in protein levels of APP-C99 in the cells treated with Numb siRNA (columns 3 and 4) as compared to control siRNA (columns 1 and 2). B. Protein levels of Numb assessed by quantifying Numb in the Western blot. Numb siRNA treatment (black bar) significantly decreases protein levels of Numb as compared to control siRNA treatment (white bar) $\left({ }^{*} \mathrm{p}<0.05\right)$, normalized to $\beta$-actin. C. APP processing assessed by quantifying ratio of APP-C99 to FL-APP in the Western blot. Numb siRNA treatment (black bar) significantly increases ratio of APP-C99 to FL-APP as compared to control siRNA treatment (white bar) $\left({ }^{*} \mathrm{P}\right.$ $<0.05$ ), normalized to $\beta$-actin. D. APP processing assessed by quantifying ratio of APP-C83 to FL-APP in the Western blot. Numb siRNA treatment (black bar) does not significantly increase ratio of APP-C83 to FL-APP as compared to control siRNA treatment (white bar), normalized to $\beta$-actin. E. Effects of RNAi knock-down of Numb on A $\beta$ levels in H4-FL-APP cells. Numb siRNA treatment (black bar) decreases A 340 levels as compared to control siRNA treatment (white bar) $\left({ }^{*} p<0.05\right.$ ). 
Next, we measured secreted $A \beta$ levels in conditioned cell culture media. 48 hours after treatment with control siRNA or Numb siRNA in H4-FL-APP cells, Numb siRNA decreased $A \beta 40$ levels: $34 \mathrm{pg} / \mathrm{ml}$ for Numb siRNA versus $48 \mathrm{pg} / \mathrm{ml}$ for control siRNA (Figure 2E, * $\mathrm{p}<0.05)$. Collectively, these data indicate that RNAi knock-down of Numb also affects APP processing and $\mathrm{A} \beta$ production in a manner similar to that of $\gamma$-secretase inhibitor treatment in H4-FL-APP cells.

As discussed in prior section, $\beta$-secretase cleaves FLAPP to produce APP-C99, which can be cleaved by $\gamma$ secretase to produce $A \beta$. Therefore, changes in APP processing and $A \beta$ production following treatments of Dab siRNA and Numb siRNA could be due to alterations in either $\beta$-secretase or $\gamma$-secretase activities. In the following experiments, we set out to determine the extent to which the alterations in APP processing and $\mathrm{A} \beta$ production following RNAi knock-down of Dab or Numb were independent of $\beta$-secretase-mediated APP processing and dependent on $\gamma$-secretase-mediated APP processing, employing $\mathrm{H} 4$ cells over-expressing APPC99 (H4-APP-C99 cells).
RNAi knock-down of Dab increased APP-CTFs levels and decreased $A \beta$ levels in H4-APP-C99 cells

We employed H4-APP-C99 cells in order to determine whether RNAi knock-down of Dab-induced alterations in APP processing and A $\beta$ levels were independent of $\beta$ secretase-mediated APP processing. As described in the prior section, APP-C99 is the product of $\beta$-secretase and harbors $\alpha$ - and $\gamma$-cleavage, but not $\beta$-cleavage sites, therefore H4-APP-C99 cells provide a valid system to assess whether any effects on APP processing are dependent on $\gamma$-secretase-mediated APP processing and independent of $\beta$-secretase-mediated APP processing.

48 hours after transfection of H4-APP-C99 cells with Dab siRNA or control siRNA, the cells were harvested and subjected to Western blot analyses in which Dab antibody was used to detect Dab. Dab immunoblotting revealed a significant reduction in protein levels of Dab in the cells treated with Dab siRNA as compared to the cells treated with control siRNA (Figure 3A). There was no significant difference in amount of $\beta$-actin in control siRNA - and Dab siRNA-treated cells. Quantification of Dab in the Western blot, normalized to $\beta$-actin, showed
A

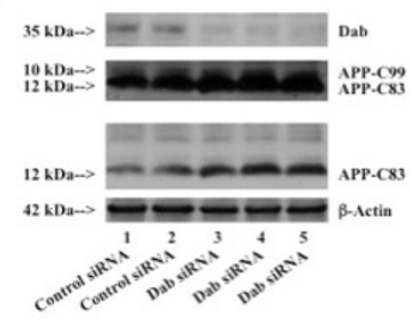

C

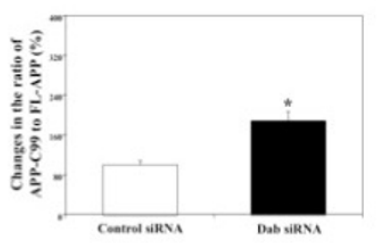

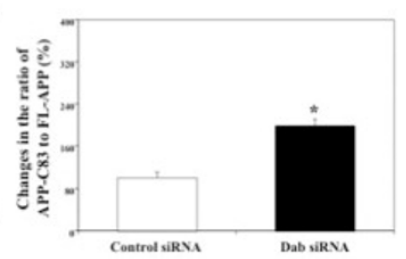

B

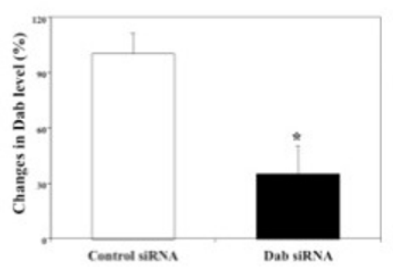

E

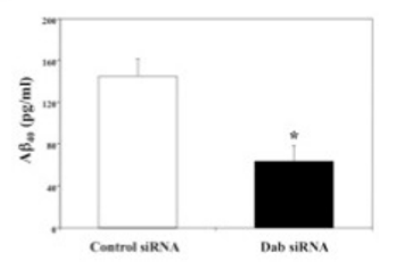

Figure 3 Effects of RNAi knock-down of Dab on APP processing and A $\boldsymbol{\beta}$ levels in H4-APP-C99 cells. In H4-APP-C99 cells, Dab siRNA treatment decreases protein levels of Dab, increases protein levels of APP-C83 and APP-C99, and decreases A $\beta$ levels. A. APP processing in Western blot analyses. Dab immunoblotting shows reductions in protein levels of Dab in the cells treated with Dab siRNA (columns 3 to 5) as compared to the cells treated with control siRNA (columns 1 and 2). APP-CTFs immunoblotting shows increases in protein levels of APP-C99 and APP-C83 in the cells treated with Dab siRNA (columns 3 to 5) as compared to control siRNA (columns 1 and 2). The blot showing the band of APP-C83 only is the same blot with less exposure time in developing the film. B. Dab protein levels assessed by quantifying Dab in the Western blot. Dab siRNA treatment significantly decreases protein levels of Dab as compared to control siRNA treatment ( ${ }^{*} p<0.05$ ), normalized to $\beta$ actin. C. APP processing assessed by quantifying ratio of APP-C99 to endogenous FL-APP in the Western blot. Dab siRNA treatment significantly increases ratio of APP-C99 to FL-APP as compared to control siRNA treatment ( $\left.{ }^{*} p<0.05\right)$, normalized to $\beta$-actin. D. APP processing assessed by quantifying ratio of APP-C83 to endogenous FL-APP in the Western blot. Dab siRNA treatment significantly increases ratio of APP-C83 to FL-APP as compared to control siRNA treatment (* $p<0.05$ ), normalized to $\beta$-actin. E. Effects of RNAi knock-down of Dab on A $\beta$ levels in H4-APP-C99 cells. Dab siRNA treatment decreases $A \beta 40$ levels as compared to control siRNA treatment $\left({ }^{*} p<0.05\right)$. 
that Dab siRNA treatment caused a $65 \%$ reduction in protein levels of Dab (Figure 3B, ${ }^{*} \mathrm{p}<0.05$ ).

We next assessed effects of RNAi-mediated knockdown of Dab on APP processing. 48 hours after transfection with Dab siRNA or control siRNA, the cells were harvested and subjected to Western blot analyses with antibody A8717. Dab siRNA treatment did not alter endogenous levels of FL-APP as compared to control siRNA treatment (data not shown). APP-CTFs immunoblotting revealed visible increases in protein levels of both APP-C99 and APP-C83 in the H4-APPC99 cells treated with Dab siRNA, compared to control siRNA (Figure 3A). There was no significant difference in amount of $\beta$-actin in the control siRNA- or Dab siRNA-treated H4-APP-C99 cells. Quantification of FLAPP, APP-C99 and APP-C83 revealed that Dab siRNA treatment led to a $188 \%$ increase in ratio of APP-C99 to FL-APP (Figure 3C, " $\mathrm{p}<0.05$ ) and a 199\% increase in ratio of APP-C83 to FL-APP (Figure 3D, " $\mathrm{p}<0.05$ ), as compared to control siRNA treatment.

We then measured secreted $A \beta$ levels in conditioned cell culture media 48 hours after treatment with either control siRNA or Dab siRNA in H4-APP-C99 cells. Dab siRNA decreased $A \beta$ levels as compared to control siRNA treatment: $63.5 \mathrm{pg} / \mathrm{ml}$ (Dab siRNA treatment), $144.5 \mathrm{pg} /$ $\mathrm{ml}$ (control siRNA treatment) (Figure 3E, ${ }^{*} \mathrm{p}<0.05$ ). These findings suggest that effects of RNAi knock-down of Dab on APP processing and A $\beta$ generation are dependent on $\gamma$-secretase- and independent of $\beta$-secretasemediated cleavage of APP.

\section{RNAi knock-down of Numb increased APP-CTFs levels and} decreased A $\beta$ levels in H4-APP-C99 cells

Next, we asked whether alterations in APP processing and $A \beta$ levels induced by RNAi knock-down of Numb were also independent of $\beta$-secretase-mediated APP processing. For this purpose, we set out to determine effects of RNAi knock-down of Numb on APP processing and A $\beta$ levels in H4-APP-C99 cells. We first established RNAi knock-down of Numb in H4-APP-C99 cells by showing that RNAi knock-down of Numb reduced protein levels of Numb in H4-APP-C99 cells (Figure 4A, B, a $46 \%$ reduction, $\left.{ }^{*} \mathrm{p}<0.05\right)$.

We then assessed effects of RNAi knock-down of Numb on levels of APP-C83, APP-C99 and A $\beta$ in H4-APP-C99 cells. 48 hours after transfection with Numb siRNA or control siRNA, the cells were harvested and subjected to Western blot analyses with antibody A8717. Numb siRNA
A

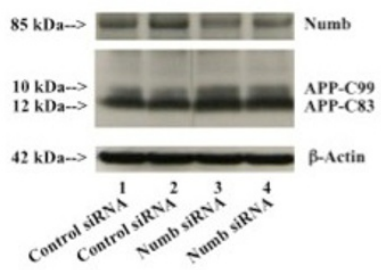

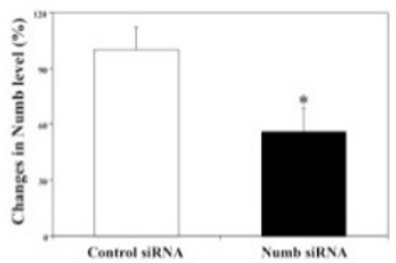

C

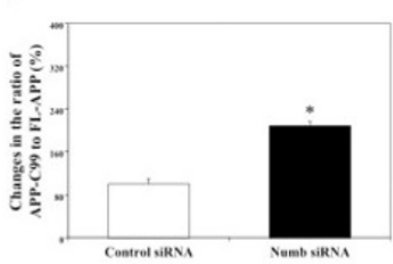

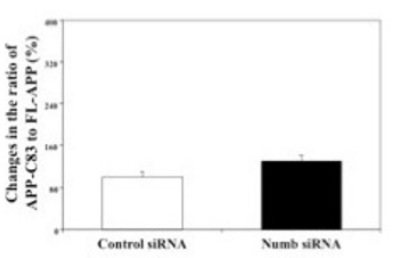

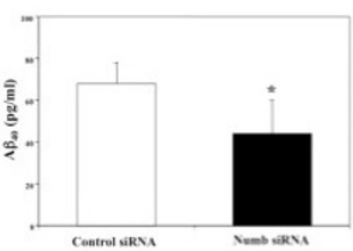

Figure 4 Effects of RNAi knock-down of Numb on APP processing and A $\boldsymbol{\beta}$ levels in H4-APP-C99 cells. In H4-APP-C99 cells, Numb siRNA treatment decreases protein levels of Numb, increases protein levels of APP-C99 and decreases A analyses. Numb immunoblotting shows reductions in protein levels of Numb in the cells treated with Numb siRNA (columns 3 and 4) as compared to control siRNA (columns 1 and 2). APP-CTFs immunoblotting shows increases in protein levels of APP-C99 in the cells treated with Numb siRNA (columns 3 and 4) as compared to control siRNA (columns 1 and 2). B. Numb protein levels assessed by quantifying Numb in the Western blot. Numb siRNA treatment significantly decreases protein levels of Numb as compared to control siRNA treatment $\left({ }^{*} p<0.05\right)$, normalized to $\beta$-actin. C. APP processing assessed by quantifying ratio of APP-C99 to endogenous FL-APP in the Western blot. Numb siRNA treatment significantly increases ratio of APP-C99 to FL-APP as compared to control siRNA treatment $\left({ }^{*} p<0.05\right)$, normalized to $\beta$-actin. D. APP processing assessed by quantifying ratio of APP-C83 to endogenous FL-APP in the Western blot. Numb siRNA treatment does not significantly increase ratio of APP-C83 to FL-APP as compared to control siRNA treatment (*p $<0.05$ ), normalized to $\beta$-actin. E. Effects of RNAi knock-down of Numb on A $\beta$ levels in H4-APP-C99 cells. Numb siRNA treatment decreases A $\beta 40$ levels as compared to control siRNA treatment (* $p<0.05$ ). 
treatment increased protein levels of both APP-C99 and APP-C83 in H4-APP-C99 cells, without altering endogenous FL-APP levels (data not shown), as compared to control siRNA treatment (Figure 4A). Quantification of the Western blot revealed that Numb siRNA treatment led to a 209\% increase in the ratio of APP-C99 to FL-APP (Figure $4 \mathrm{C},{ }^{*} \mathrm{p}<0.05$ ) and only a $130 \%$ increase in ratio of APP-C83 to FL-APP (Figure 3D, N.S.), as compared to control siRNA treatment. Numb siRNA treatment decreased secreted $A \beta$ levels as compared to control siRNA treatment in H4-APP-C99 cells: $44 \mathrm{pg} / \mathrm{ml}$ (Numb siRNA treatment) versus $68 \mathrm{pg} / \mathrm{ml}$ (control siRNA treatment) (Figure 4E, ${ }^{*} \mathrm{p}<0.05$ ). These findings suggest that RNAi knock-down of Numb may affect APP processing and $A \beta$ production at least partially by inhibiting $\gamma$-secretase-, but not $\beta$-secretase-, mediated cleavage of APP, a manner similar to that of $\gamma$-secretase inhibitors.

\section{RNAi knock-down of APP decreased Numb levels in H4- APP cells}

A recent study by Chapman et al. [24] showed that high levels of Notch can decrease Numb and Numblike. Both APP and Notch are substrates of $\gamma$-secretase, we therefore assessed effects of APP on Numb levels in H4APP cells. 48 hours after transfection with APP siRNA or control siRNA, the cells were harvested and subjected to Western blot analyses with antibodies anti-Numb and A8717. We first showed that APP siRNA treatment decreased protein levels of both FL-APP and APP-CTFs (Figure 5A), suggesting that the APP siRNA treatment can reduce levels of APP (both FL-APP and APP-CTFs) in H4-APP cells. Then we were able to show that the same APP siRNA treatment also reduced levels of Numb in H4-APP cells (Figure 5A). Quantification of the Western blot revealed that APP siRNA treatment led to a $55 \%$ (Figure 5B, **p < 0.01), 41\% (Figure 5C, **p $<0.01$ ) and $25 \%$ (Figure $5 \mathrm{D}, * \mathrm{p}<0.01$ ) reduction in levels of FL-APP, APP-CTFs and Numb, respectively, as compared to control siRNA treatment. These findings suggest that RNAi knock-down of APP may affect Numb metabolism as well.

\section{Control siRNA affected neither APP processing nor A $\beta$ levels in H4-FL-APP or H4-APP-C99 cells}

Finally, We found that control siRNA did not affect APP processing or alter $A \beta$ levels as compared to saline
A

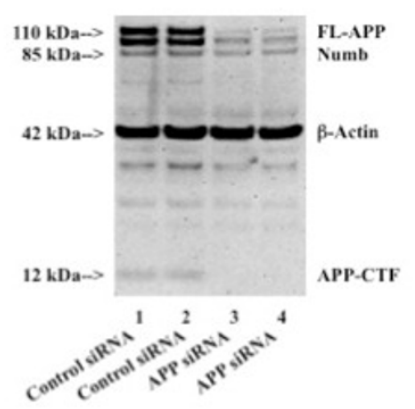

C

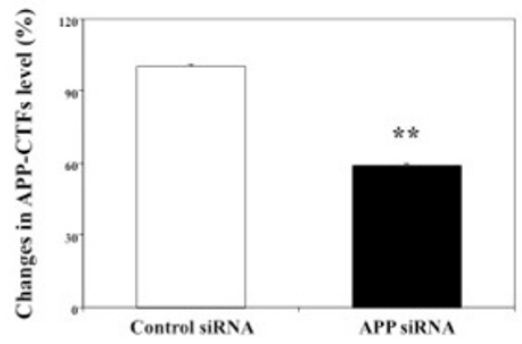

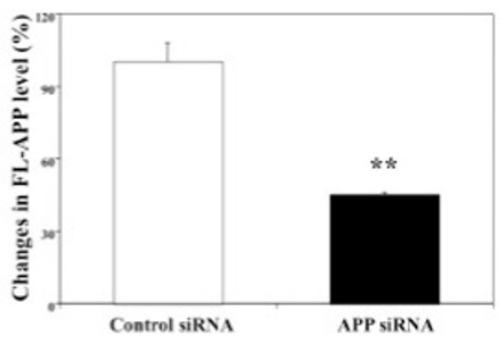

D

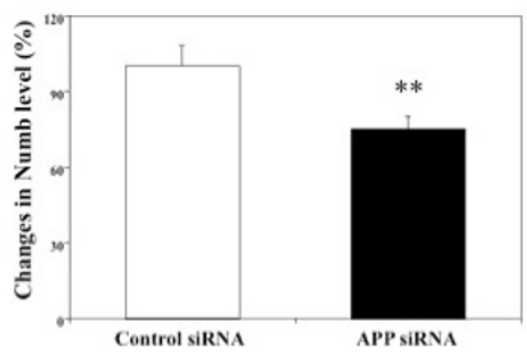

Figure 5 Effects of RNAi knock-down of APP on levels of Numb in H4-APP cells. In H4-APP cells, APP siRNA treatment decreases protein levels of Numb. A. Levels of FL-APP, APP-CTFs and Numb in Western blot analyses. APP immunoblotting shows reductions in protein levels of FL-APP, APP-CTFs in the cells treated with APP siRNA (columns 3 and 4) as compared to that treated with control siRNA (columns 1 and 2). Numb immunoblotting shows reductions in protein levels of Numb in the cells treated with Numb siRNA (columns 3 and 4 ) as compared to control siRNA (columns 1 and 2). B. FL-APP levels assessed by quantifying FL-APP in the Western blot. APP siRNA treatment significantly decreases protein levels of FL-APP as compared to control siRNA (** $p<0.01$ ), normalized to $\beta$-actin. C. APP-CTFs levels assessed by quantifying APP-CTFs in the Western blot. APP siRNA treatment significantly decreases protein levels of APP-CTFs as compared to control siRNA $(* * p<0.01)$, normalized to $\beta$-actin. D. Numb protein levels assessed by quantifying Numb in the Western blot. APP siRNA treatment significantly decreases protein levels of Numb as compared to control siRNA treatment $\left({ }^{*} p<0.05\right)$, normalized to $\beta$-actin. 
treatment in H4-FL-APP cells or H4-APP-C99 cells (data not shown). These results confirmed that effects of RNAi knock-down of Dab or Numb on APP processing and $A \beta$ levels in our experiments were not due to control siRNA (scrambled siRNA) effects, but owing to reductions in protein levels of Dab and Numb.

\section{Discussion}

$\mathrm{A} \beta$ accumulation resulting from imbalance between $A \beta$ generation and clearance in brain is a foundation of $A D$ neuropathogenesis [[1], see review in [26]]. A $\beta$ is produced via serial proteolysis of APP by two proteases, $\beta$ secretase and $\gamma$-secretase [5-8]. Several APP adaptor proteins [see review in [15]] have previously been shown to affect APP processing and $A \beta$ production following overexpression [27-35]. Recent studies showed that RNAi-mediated knock-down of X11 $\alpha$, ShcC, Fe65 and ARH can also affect APP processing and A $\beta$ levels $[16,17,36]$. However, effects of RNAi knock-down of other APP adaptor proteins, including Dab and Numb, on APP processing and A $\beta$ production have not been previously reported. Here, we show for the first time that RNAi knock-down of Dab and Numb significantly affects APP processing and A $\beta$ levels.

RNAi knock-down of Dab and Numb caused increases in levels of APP-C99 and APP-C83 in absence of alterations in FL-APP levels, and RNAi for Dab and Numb decreased secreted $A \beta$ levels in H4-FL-APP cells. As described in prior section, $\beta$-secretase and $\alpha$-secretase cleaves FL-APP to produce APP-C99 and APP-C83 respectively, then $\gamma$-secretase cleaves APP-C99 and APP$\mathrm{C} 83$ to generate $\mathrm{A} \beta$ and $\mathrm{p} 3$ respectively, and AICD. Therefore, the observed increases in APP-C99 and APPC83 levels following Dab or Numb siRNA treatment could be due either to increases in the activities of $\beta$ secretase and/or $\alpha$-secretase, or to decreases in $\gamma$-secretase cleavage of APP-C99 and C83. To distinguish these two possibilities, we repeated RNAi knock-down of Dab and Numb experiment in H4 cells over-expressing APPC99 (H4-APP-C99 cells), the $\beta$-secretase cleavage product of APP, and found that Dab or Numb siRNA treatment still increased protein levels of APP-C99 and APP$\mathrm{C} 83$ and decreased levels of secreted $\mathrm{A} \beta$. These data indicate that the observed changes in APP processing and $A \beta$ levels following RNAi knock-down of Dab and Numb are independent of $\beta$-secretase-, and most likely are due to inhibition of $\gamma$-secretase-mediated cleavage of APP.

One possible explanation for our current results is that Dab and Numb, APP adaptor proteins, are essential for $\gamma$-secretase cleavage of APP-C99 to generate A $\beta$, perhaps via facilitating APP trafficking to the sites where $\gamma$ secretase can cleave it. Reductions in levels of Dab and Numb by RNAi knock-down for their encoded genes,
$D A B$ and $N U M B$, will cause a "break" in APP trafficking to the sites where $\gamma$-secretase is located, thereby leading to the accumulation of APP-CTFs (APP-C99 and APPC83), the substrates of $\gamma$-secretase, and reduction in levels of $A \beta$, the products of $\gamma$-secretase. In the future studies, we will assess effects of over-expression and reduction of Dab and Numb on APP trafficking to further test this hypothesis.

To date, many other substrates have been reported to undergo $\gamma$-secretase cleavage in addition to APP: Notch, ErbB-4, E-cadherin, the LDL receptor-related protein (LRP), CD44 and nectin-1- $\alpha$ [37-43]. All of these substrates are type I membrane proteins residing at or near cell surface, which undergo ectodomain shedding prior to $\gamma$-secretase-like cleavage and release ICDs (intracellular domains) following proteolysis. In our future research, we will determine whether or not RNAi knock-down of Dab and Numb can affect processing of these other $\gamma$-secretase substrates. It is also important to determine the potential interactions between Notch signaling with APP adaptor proteins including Numb, Dab, $\mathrm{X} 11 \alpha$, ShcC and Fe65 in our established cellular model.

Taking together, our findings demonstrate, for the first time, that RNAi-mediated knock-down of Dab and Numb decrease secretion of $A \beta$, conceivably via inhibiting $\gamma$-secretase cleavage of APP. These data, together with those of previous studies, imply that blocking interaction of APP with either Dab or Numb, and perhaps other APP adaptor proteins (e.g., X11 $\alpha$, ShcC and Fe65), could be a novel therapeutical strategy for treating and/ or preventing $\mathrm{AD}$ by lowering $\mathrm{A} \beta$ accumulation.

Increasing evidence suggest a role of Notch signaling pathway in neurodegeneration of adult vertebrate nervous system and in AD neuropathogenesis [44,45]. A recent study showed that overexpressions of APP, APLP1 and APLP2 induce Notch gain-of-function phenotypes in Drosophila, suggesting a cross-talk between APP family and Notch [46]. Numb and Dab have been suggested to be mediators of such APP and Notch interactions [46]. Moreover, it has been suggested that pharmacological processing of APP in AD treatment may cause alterations in Notch phenotype through Dab and Numb, thereby contributing to the side effects of the treatment or even to increasing risk of AD $[46,47]$. Therefore, Dab and Numb could be important targets in the development of therapeutical strategies for AD. Studies to further identify the roles of Dab and Numb in AD neuropathogenesis, including assessment of effects of Dab and Numb on APP processing, A $\beta$ accumulation, synaptic function and apoptosis, are warranted in the future.

A recent study [24] showed that at low levels of Notch signaling, Numb and Numblike can negatively regulate Notch, however, high levels Notch can reduce protein levels of Numb and Numblike. These findings suggest 
that a reciprocal negative regulation between Notch and Numb/Numblike. In the present study, we have illustrated for the first time that reductions in APP levels can lead to reductions in Numb levels, and reductions in Numb levels can lead to reductions in $A \beta$ levels. Collectively, these findings suggest that APP (and A $\beta$ ) and Numb can also have a reciprocal negative regulation, leading to reductions in $A \beta$ levels.

In conclusion, RNAi-mediated knock-down of APP adaptor proteins, Dab and Numb, attenuate $\gamma$-secretasemediated cleavage of APP, leading to decreased A $\beta$ levels. Our findings, together with those of previous studies, suggest that pharmaceutical modulation of APP adaptor proteins, might potentially serve as a novel therapeutic approach to treating and preventing $\mathrm{AD}$. The notion of attenuating $\gamma$-secretase cleavage of APP via APP adaptor proteins, such as $\mathrm{X} 11 \alpha$, ShcC, Fe65, Dab and Numb, is particularly attractive, because interactions with these APP adaptor proteins may bypass unwanted effects of $\gamma$-secretase inhibition owing to impaired proteolysis of other $\gamma$ secretase substrates. More studies are needed to further investigate these findings and to assess feasibility of such a therapeutic strategy aimed at lowering $A \beta$ levels.

\section{Abbreviations}

AD: Alzheimer's disease; siRNA: Small interfering RNA; RNAi: RNA interference; ANOVA: Analysis of variance; NTF: Amino-terminal fragment; CTF: Carboxylterminal fragment; FL: Full-length; APP: Amyloid precursor protein; $A \beta$ : Amyloid- $\beta$ protein; PTB: Phosphotyrosine binding.

\section{Acknowledgements}

This research was supported by K08NS048140, R21AG029856, R21AG038994 and R01 GM088801 (National Institutes of Health), USA, Jahnigen Career Development Award (American Geriatrics Society), USA; Investigator Initiated Research Grant (Alzheimer's Association), Cure Alzheimer's Fund, USA (to Z. X.). MH 60009 (National Institute of Mental Health), USA, Cure Alzheimer's Fund (to R.T.).

\section{Author details}

'Department of Neurology, Genetics and Aging Research Unit, MassGeneral Institute for Neurodegenerative Disease, Massachusetts General Hospital and Harvard Medical School, Charlestown, MA 02129-2060, USA. ²Department of Anesthesia and Critical Care, Geriatric Anesthesia Research Unit,

Massachusetts General Hospital and Harvard Medical School, Charlestown, MA 02129-2060, USA. ${ }^{3}$ Center for Neurological Diseases, Harvard Institute of Medicine and Harvard Medical School, Boston, MA 02115, USA. ${ }^{4}$ Graduate student, Department of Psychology, University of Southern California, Los Angeles, CA 90089, USA.

\section{Authors' contributions}

ZX carried out RNAi interference (RNAi) studies, Western blot analysis, experimental design, data analysis and wrote the manuscript. YD carried out RNAi studies and Western blot analysis. UM carried out cell culture and Western blot studies. WX carried out ELISA measurement of A levels. RT carried out the data analysis and drafted the manuscript. All authors read and approved the final manuscript.

\section{Competing interests}

The authors declare that they have no competing interests.

Received: 25 December 2011 Accepted: 22 March 2012 Published: 22 March 2012

\section{References}

1. Glenner GG, Wong CW: Alzheimer's disease and Down's syndrome: sharing of a unique cerebrovascular amyloid fibril protein. Biochem Biophys Res Commun 1984, 122:1131-1135.

2. Goate A, Chartier-Harlin MC, Mullan M, Brown J, Crawford F, Fidani L, Giuffra L, Haynes A, Irving N, James L, et al: Segregation of a missense mutation in the amyloid precursor protein gene with familial Alzheimer's disease. Nature 1991, 349:704-706

3. Masters $\mathrm{CL}$, Simms G, Weinman NA, Multhaup G, McDonald BL, Beyreuther K: Amyloid plaque core protein in Alzheimer disease and Down syndrome. Proc Natl Acad Sci USA 1985, 82:4245-4249.

4. Selkoe DJ, Podlisny MB, Joachim CL, Vickers EA, Lee G, Fritz LC, Oltersdorf T: Beta-amyloid precursor protein of Alzheimer disease occurs as 110- to 135-kilodalton membrane-associated proteins in neural and nonneural tissues. Proc Natl Acad Sci USA 1988, 85:7341-7345

5. Hussain I, Powell D, Howlett DR, Tew DG, Meek TD, Chapman C, Gloger IS, Murphy KE, Southan CD, Ryan DM, et al: Identification of a novel aspartic protease (Asp 2) as beta-secretase. Mol Cell Neurosci 1999, 14:419-427.

6. Sinha S, Lieberburg I: Cellular mechanisms of beta-amyloid production and secretion. Proc Natl Acad Sci USA 1999, 96:11049-11053.

7. Vassar R, Bennett BD, Babu-Khan S, Kahn S, Mendiaz EA, Denis P, Teplow DB, Ross S, Amarante P, Loeloff R, et al: Beta-secretase cleavage of Alzheimer's amyloid precursor protein by the transmembrane aspartic protease BACE. Science 1999, 286:735-741.

8. Yan R, Bienkowski MJ, Shuck ME, Miao H, Tory MC, Pauley AM, Brashier JR, Stratman NC, Mathews WR, Buhl AE, et al: Membrane-anchored aspartyl protease with Alzheimer's disease beta-secretase activity. Nature 1999, 402:533-537.

9. Gu Y, Misonou H, Sato T, Dohmae N, Takio K, Ihara Y: Distinct intramembrane cleavage of the beta-amyloid precursor protein family resembling gamma-secretase-like cleavage of Notch. J Biol Chem 2001, 276:35235-35238.

10. Sastre M, Steiner H, Fuchs K, Capell A, Multhaup G, Condron MM, Teplow DB, Haass C: Presenilin-dependent gamma-secretase processing of beta-amyloid precursor protein at a site corresponding to the S3 cleavage of Notch. EMBO Rep 2001, 2:835-841.

11. Yu C, Kim SH, Ikeuchi T, Xu H, Gasparini L, Wang R, Sisodia SS: Characterization of a presenilin-mediated amyloid precursor protein carboxyl-terminal fragment gamma. Evidence for distinct mechanisms involved in gamma -secretase processing of the APP and Notch1 transmembrane domains. J Biol Chem 2001, 276:43756-43760.

12. Esch FS, Keim PS, Beattie EC, Blacher RW, Culwell AR, Oltersdorf T, McClure D, Ward PJ: Cleavage of amyloid beta peptide during constitutive processing of its precursor. Science 1990, 248:1122-1124

13. Sisodia SS, Koo EH, Beyreuther K, Unterbeck A, Price DL: Evidence that beta-amyloid protein in Alzheimer's disease is not derived by normal processing. Science 1990, 248:492-495.

14. Wolfe MS: The secretases of Alzheimer's disease. Curr Top Dev Biol 2003, 54:233-261.

15. King GD, Scott Turner R: Adaptor protein interactions: modulators of amyloid precursor protein metabolism and Alzheimer's disease risk? Exp Neurol 2004, 185:208-219.

16. Xie Z, Dong Y, Maeda U, Xia W, Tanzi RE: RNA interference silencing of the adaptor molecules ShcC and Fe65 differentially affect amyloid precursor protein processing and Abeta generation. J Biol Chem 2007, 282:4318-4325.

17. Xie Z, Romano DM, Tanzi RE: RNA interference-mediated silencing of $\mathrm{X} 11$ alpha and $\mathrm{X} 11$ beta attenuates amyloid beta-protein levels via differential effects on beta-amyloid precursor protein processing. J Biol Chem 2005, 280:15413-15421.

18. Howell BW, Lanier LM, Frank R, Gertler FB, Cooper JA: The disabled 1 phosphotyrosine-binding domain binds to the internalization signals of transmembrane glycoproteins and to phospholipids. Mol Cell Biol 1999, 19:5179-5188.

19. Trommsdorff M, Borg JP, Margolis B, Herz J: Interaction of cytosolic adaptor proteins with neuronal apolipoprotein $E$ receptors and the amyloid precursor protein. J Biol Chem 1998, 273:33556-33560.

20. Homayouni R, Rice DS, Sheldon M, Curran T: Disabled-1 binds to the cytoplasmic domain of amyloid precursor-like protein 1. J Neurosci 1999, 19:7507-7515. 
21. Yun M, Keshvara L, Park CG, Zhang YM, Dickerson JB, Zheng J, Rock CO, Curran T, Park HW: Crystal structures of the Dab homology domains of mouse disabled 1 and 2. J Biol Chem 2003, 278:36572-36581.

22. Roncarati $R$, Sestan N, Scheinfeld MH, Berechid BE, Lopez PA, Meucci O, McGlade JC, Rakic P, D'Adamio L: The gamma-secretase-generated intracellular domain of beta-amyloid precursor protein binds Numb and inhibits Notch signaling. Proc Natl Acad Sci USA 2002, 99:7102-7107.

23. Zhong $W$, Jiang $M M$, Weinmaster $G$, Jan $L Y$, Jan $Y N$ : Differential expression of mammalian Numb, Numblike and Notch1 suggests distinct roles during mouse cortical neurogenesis. Development 1997, 124:1887-1897.

24. Chapman G, Liu L, Sahlgren C, Dahlqvist C, Lendahl U: High levels of Notch signaling down-regulate Numb and Numblike. J Cell Biol 2006, 175:535-540.

25. Xie Z, Moir RD, Romano DM, Tesco G, Kovacs DM, Tanzi RE: Hypocapnia induces caspase- 3 activation and increases abeta production. Neurodegener Dis 2004, 1:29-37.

26. Tanzi RE, Bertram L: Twenty years of the Alzheimer's disease amyloid hypothesis: a genetic perspective. Cell 2005, 120:545-555.

27. Biederer T, Cao X, Sudhof TC, Liu X: Regulation of APP-dependent transcription complexes by Mint/X11s: differential functions of Mint isoforms. J Neurosci 2002, 22:7340-7351.

28. Borg JP, Yang Y, De Taddeo-Borg M, Margolis B, Turner RS: The X11alpha protein slows cellular amyloid precursor protein processing and reduces Abeta40 and Abeta42 secretion. J Biol Chem 1998, 273:14761-14766.

29. Guenette S, Chang Y, Hiesberger T, Richardson JA, Eckman CB, Eckman EA Hammer RE, Herz J: Essential roles for the FE65 amyloid precursor protein-interacting proteins in brain development. EMBO J 2006, 25:420-431.

30. Guenette SY, Chen J, Ferland A, Haass C, Capell A, Tanzi RE: hFE65L influences amyloid precursor protein maturation and secretion. $J$ Neurochem 1999, 73:985-993.

31. King GD, Cherian K, Turner RS: X11alpha impairs gamma- but not betacleavage of amyloid precursor protein. J Neurochem 2004, 88:971-982.

32. King GD, Perez RG, Steinhilb ML, Gaut JR, Turner RS: X11alpha modulates secretory and endocytic trafficking and metabolism of amyloid precursor protein: mutational analysis of the YENPTY sequence. Neuroscience 2003, 120:143-154.

33. Lee JH, Lau KF, Perkinton MS, Standen CL, Shemilt SJ, Mercken L, Cooper JD, McLoughlin DM, Miller CC: The neuronal adaptor protein $\mathrm{X} 11$ alpha reduces Abeta levels in the brains of Alzheimer's APPswe Tg2576 transgenic mice. J Biol Chem 2003, 278:47025-47029.

34. McLoughlin DM, Irving NG, Brownlees J, Brion JP, Leroy K, Miller CC: Mint2/ X11-like colocalizes with the Alzheimer's disease amyloid precursor protein and is associated with neuritic plaques in Alzheimer's disease. Eur J Neurosci 1999, 11:1988-1994.

35. Sastre M, Turner RS, Levy E: X11 interaction with beta-amyloid precursor protein modulates its cellular stabilization and reduces amyloid betaprotein secretion. J Biol Chem 1998, 273:22351-22357.

36. Noviello C, Vito P, Lopez P, Abdallah M, D'Adamio L: Autosomal recessive hypercholesterolemia protein interacts with and regulates the cell surface level of Alzheimer's amyloid beta precursor protein. J Biol Chem 2003, 278:31843-31847.

37. De Strooper B, Annaert W, Cupers P, Saftig P, Craessaerts K, Mumm JS, Schroeter EH, Schrijvers $V$, Wolfe MS, Ray WJ, et al: A presenilin-1dependent gamma-secretase-like protease mediates release of Notch intracellular domain. Nature 1999, 398:518-522.

38. Kim DY, Ingano LA, Kovacs DM: Nectin-1alpha, an immunoglobulin-like receptor involved in the formation of synapses, is a substrate for presenilin/gamma-secretase-like cleavage. J Biol Chem 2002, 277:49976-49981.

39. Lammich S, Okochi M, Takeda M, Kaether C, Capell A, Zimmer AK, Edbauer D, Walter J, Steiner H, Haass C: Presenilin-dependent intramembrane proteolysis of CD44 leads to the liberation of its intracellular domain and the secretion of an Abeta-like peptide. $J$ Biol Chem 2002, 277:44754-44759.

40. Marambaud P, Shioi J, Serban G, Georgakopoulos A, Sarner S, Nagy V, Baki L, Wen P, Efthimiopoulos S, Shao Z, et al: A presenilin-1/gammasecretase cleavage releases the E-cadherin intracellular domain and regulates disassembly of adherens junctions. EMBO J 2002, 21:1948-1956.
41. May P, Reddy YK, Herz J: Proteolytic processing of low density lipoprotein receptor-related protein mediates regulated release of its intracellular domain. J Biol Chem 2002, 277:18736-18743.

42. Ni CY, Murphy MP, Golde TE, Carpenter G: gamma -Secretase cleavage and nuclear localization of ErbB-4 receptor tyrosine kinase. Science 2001, 294:2179-2181.

43. Wong PC, Zheng $\mathrm{H}$, Chen $\mathrm{H}$, Becher MW, Sirinathsinghji DJ, Trumbauer ME, Chen HY, Price DL, Van der Ploeg LH, Sisodia SS: Presenilin 1 is required for Notch1 and DII1 expression in the paraxial mesoderm. Nature 1997, 387:288-292

44. Gaiano N, Fishell G: The role of notch in promoting glial and neural stem cell fates. Annu Rev Neurosci 2002, 25:471-490.

45. Selkoe D, Kopan R: Notch and Presenilin: regulated intramembrane proteolysis links development and degeneration. Annu Rev Neurosci 2003, 26:565-597.

46. Merdes G, Soba P, Loewer A, Bilic MV, Beyreuther K, Paro R: Interference of human and Drosophila APP and APP-like proteins with PNS development in Drosophila. EMBO J 2004, 23:4082-4095.

47. Turner PR, O'Connor K, Tate WP, Abraham WC: Roles of amyloid precursor protein and its fragments in regulating neural activity, plasticity and memory. Prog Neurobiol 2003, 70:1-32.

doi:10.1186/2047-9158-1-8

Cite this article as: Xie et al:: RNAi-mediated knock-down of Dab and Numb attenuate A $\beta$ levels via $\gamma$-secretase mediated APP processing. Translational Neurodegeneration 2012 1:8.

\section{Submit your next manuscript to BioMed Central and take full advantage of:}

- Convenient online submission

- Thorough peer review

- No space constraints or color figure charges

- Immediate publication on acceptance

- Inclusion in PubMed, CAS, Scopus and Google Scholar

- Research which is freely available for redistribution

Submit your manuscript at www.biomedcentral.com/submit
C) Biomed Central 